\title{
A measure of competitive access to destinations for comparing across multiple study regions
}

3 Jeff Allen

4 Department of Geography and Planning, University of Toronto St. George

5100 St. George St., Toronto, Ontario M5S 3G3, Canada

6 jeff.allen@utoronto.ca

7 Steven Farber

8 Department of Human Geography, University of Toronto Scarborough

91265 Military Trail, Toronto, Ontario, M1C 1A4, Canada

10 steven.farber@utoronto.ca

11 (02019. This manuscript preprint version is made available under the CC-BY-NC-ND 4.0 license

12 http://creativecommons.org/licenses/by-nc-nd/4.0/

13 The final published version in Geographical Analysis can be found at https://doi.org/10.1111/

14 gean. 12188

\section{Abstract}

Accessibility is now a common way to measure the benefits provided by transportation-land use systems. Despite its widespread use, few measurement options allow for the comparison of accessibility across multiple urban systems, and most do not adequately control for market competition between demand-side actors and supply-side facilities in localized markets. In this paper we develop a measure of competitive access to destinations that can be used to accurately compare accessibility between regions. This measure stems from spatial interaction modelling and accounts for competition at both the supply and demand sides of analysis, regional differences in transportation networks and travel behaviour, and any imbalance between the size of the population and the number of opportunities. We use this method to compute access to employment for Canada's eight largest cities to comparatively examine inequalities in accessibility, both within and between cities, and by travel mode. 


\section{$1 \quad 1$ Introduction}

2 Accessibility, from an urban geography perspective, is typically understood as the potential for interaction or ease of reaching destinations (Hansen, 1959). Accessibility is a function of transport networks, land use characteristics (e.g. one's location in relation to the distribution of destinations), as well as individual social and economic factors (e.g. can someone afford a car) (Handy \& Niemeier, 1997; Kwan, 1998; Geurs \& Van Wee, 2004). Accessibility measures have been used in a wide range of studies analyzing their effect on activity participation rates (e.g. Paez et al., 2009), employment outcomes (e.g. Merlin \& Hu, 2017), commuting times (e.g. Kawabata \& Shen, 2007), as well as in normative studies analyzing inequalities between neighbourhoods and population groups (e.g. Delbosc \& Currie, 2011), examining changes in accessibility over time (e.g. Farber \& Fu, 2017), or comparing levels of access by different travel modes (e.g. Benenson et al., 2011).

Despite the quantity of research on accessibility, there are only a few studies that compare accessibility between different cities. One reason for this is the difficulty in generating accessibility metrics which can be used to meaningfully compare between regions which have different quantities and distributions of populations, opportunities, and transport networks. Existing multi-city studies tend to use non-competitive measures (Kawabata \& Shen, 2006; Grengs et al., 2010; Levine et al., 2012; Owen \& Levinson, 2014; Deboosere \& El-Geneidy, 2018), which sum the number of opportunities (e.g. jobs) that can be reached from a location. However, the raw values of accessibility computed for locations in one region are not a meaningful comparator to access scores in another region in situations where there are capacity constraints at the destination (e.g. like access to employment where each job can only be filled by one worker). This is because the accumulation of supply is not adequately discounted for the amount of demand it is servicing. For example, central Toronto may have tenfold the amount of nearby jobs than central Winnipeg, but if the nearby labour force is ten times the size, then access should be approximately equivalent as there is an equal number of accessible jobs per worker.

Accordingly, the objective of this paper is to develop a measure of access to destinations that accounts for competition and can be used to compare between regions. Specifically, this measure accounts for competition at both the supply and demand sides of analysis, similar to the balancing factors of a doubly-constrained spatial interaction model (e.g. Geurs \& van Eck, 2003; Horner, 2004). It also accounts for regional specific transportation networks and travel behaviour as well as differing imbalances between the size of the population and the number of available opportunities. We apply this method to computing access to employment for Canada's eight largest urban regions. We exemplify its use by analyzing spatial inequalities of accessibility within and between regions as well as by travel mode. The data and methods used are all open-source, so they can be shared and replicated with minimal cost (https://github.com/SAUSy-Lab/canada-transit-access). 


\section{Competitive Accessibility}

2 At a basic level, measuring accessibility is concerned with evaluating how well a city's land use and transportation system provides people with the opportunity to travel to a broad spectrum of destinations in a reasonable amount of time. Methodologically, there are a number of ways in which accessibility has been measured in research and practice (Handy \& Niemeier, 1997; Geurs \& Van Wee, 2004). Accessibility measures are typically either place-based (linked to an area or a specific point in space) or person-based (linked to an individual, often through their daily activity patterns) (Miller, 2007). Probably the most common form of measuring place-based access to destination metrics are integral, they sum opportunities that can be reached from specific location(s) in space (Handy \& Niemeier, 1997; Kwan, 1998). These are typically formulated as follows:

$$
A_{i}=\sum_{j=1}^{J} O_{j} f\left(t_{i, j}\right)
$$

Where $A_{i}$ is the measure of access for a location $i . O_{j}$ is the number of opportunities at a location $j . O_{j}$ can be interpreted as the attractiveness, or gravitational pull, at location $j . f\left(t_{i, j}\right)$ is a decreasing function of travel cost, $t$, from $i$ to $j . t_{i, j}$ is based on one or more impedance factors like travel time or monetary cost. The simplest form of $f\left(t_{i, j}\right)$ is a threshold indicator, which returns a 0 or 1 whether or not the travel time is less than a threshold. In this case, $A_{i}$ is interpreted as the number of opportunities (e.g. jobs) that can be reached within a set travel time (e.g. within 30 minutes). Gravity models extend this by using a decay function to weight nearby destinations more than destinations that are further away. However, measures computed by (1) are most suitable for analyzing access to destinations where there is no competition for resources at the destination (i.e. for situations where being able to access an opportunity is not dependent on other people accessing it as well).

Equation (1) can be expanded in order to incorporate competition for resources at the destination (Weibull, 1976). This has been commonly used in measuring access to health services, often formulated as floating-catchment approaches, to output accessibility measures as intuitive metrics like doctors per person (Luo \& Wang, 2003; Delamater, 2013). Applied to access to employment, competitive measures can account for how employment opportunities and the labour force are both spatially distributed and overlapping, and that competition exists among the labour force for jobs (Shen, 1998; Geurs \& van Eck, 2003; Kawabata \& Shen, 2006). Mathematically, this involves normalizing opportunities at $j$ by the population within their catchment area, $L_{j}$.

$$
A_{i}=\sum_{j=1}^{J} \frac{O_{j} f\left(t_{i, j}\right)}{L_{j}} \quad L_{j}=\sum_{i=1}^{I} P_{i} f\left(t_{i, j}\right)
$$

Where $P_{i}$ is the population at $i$ competing for opportunities. In research on access to health services, this metric has been simplified by setting $f\left(t_{i, j}\right)$ to an indicator function to generate population to 
provider ratios (these are commonly referred to as 2-step floating catchment area measures) (Luo \& Wang, 2003; Delamater, 2013).

This can be expanded to account for competition at both the origin and destination locations by incorporating $A_{i}$ into the equation for $L_{j}$ to normalize for the number of opportunities that someone at location, $i$, can reach.

$$
A_{i}=\sum_{j=1}^{J} \frac{O_{j} f\left(t_{i, j}\right)}{L_{j}} \quad L_{j}=\sum_{i=1}^{I} \frac{P_{i} f\left(t_{i, j}\right)}{A_{i}}
$$

This form is akin to a doubly constrained spatial interaction model, where balancing factors are used to ensure that the sum of flows from $i$ and destined to $j$ equals the observed amount arriving and departing from each zone (Wilson, 1971; Fotheringham \& O'Kelly, 1989). $A_{i}$ and $L_{j}$ are simply the inverse of the balancing factors in the doubly constrained model. Since $L_{j}$ and $A_{i}$ are mutually dependent, they have to be estimated iteratively until they reach convergence. Convergence is guaranteed if $\sum O j=\sum P i$ (e.g. if the labour force is equal to the number of employment opportunities). Figure 1 shows, for a simplistic linear city, how the measure of competitive accessibility in (3) converges after several iterations.

Figure 1: Iterative convergence of competitive accessibility for a simple linear city

\begin{tabular}{|c|c|c|c|c|}
\hline$a$ & $x$ & $b$ & $y$ & $c$ \\
0 & 0 & 0 & -0 & $C$ \\
$P_{a}=500$ & $O_{x}=700$ & $P_{b}=600$ & $O_{y}=800$ & $P_{c}=400$
\end{tabular}

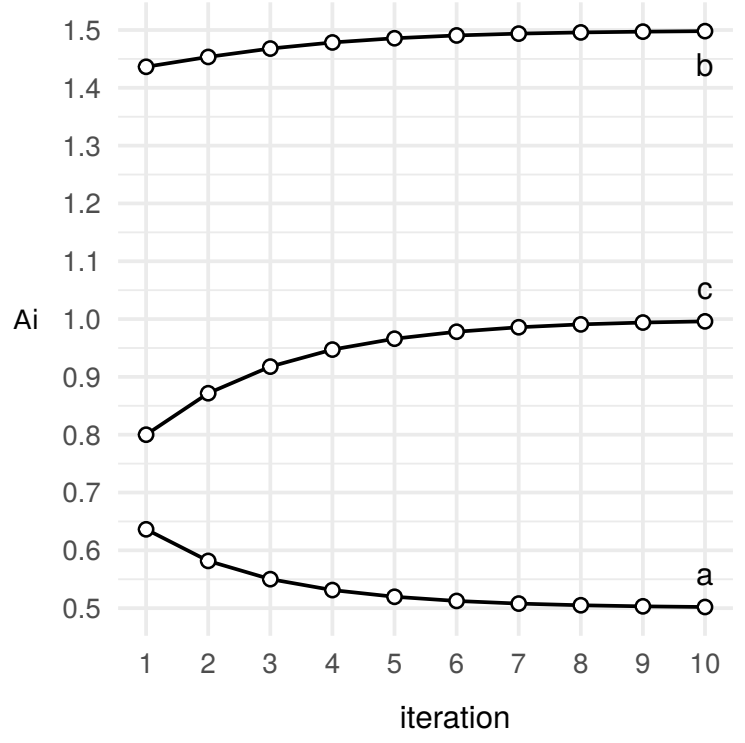
compete for workers who have varying levels of access to jobs, and people compete for jobs at 
locations which have varying levels of access to the labour force (Geurs \& van Eck, 2003; Horner, 2004; Merlin \& Hu, 2017). This type of measure has been applied at a regional scale in Sardinia (De Montis et al., 2011; Caschili et al., 2015), Sweden (Östh, 2011; Östh et al., 2016), and the Netherlands (Geurs \& van Eck, 2003) as well as at an urban scale in Montreal (Cerda, 2009; ElGeneidy \& Levinson, 2011) and Los Angeles (Merlin \& Hu, 2017). These studies have shown that competitive accessibility measures are strongly correlated with non-competitive measures; however, they have differing spatial distributions and rank orders, which can impact conclusions and specific policy recommendations. Merlin and $\mathrm{Hu}$ (2017) also showed that competitive measures of access to jobs are a better predictor of employment outcomes than integral measures which do not consider competition. Despite these few existing studies, the majority of research on access to employment does not consider competition effects or only considers competition at the destination (i.e. a two step approach). Up until recently, this is likely due to the computational effort of iterative solutions in regions with many origins and destinations. Moreover, the majority of existing studies comparing accessibility between regions do not consider competition effects (Kawabata \& Shen, 2006; Grengs et al., 2010; Levine et al., 2012; Owen \& Levinson, 2014; Deboosere \& El-Geneidy, 2018). From our knowledge, only Horner (2004) has used a doubly constrained approach to compare accessibility between regions (for 10 cities in the United States). The study by Horner (2004) only used distance as impedance rather than mode-specific travel times and it did not consider unemployed populations competing for jobs, despite the fact that these variables can differ between regions.

Accordingly, we expand upon the measure of competitive accessibility shown in equation (3) in order to account for regions with different levels of imbalance between origin-constraints (e.g the size of labour force) and destination-constraints (e.g the number of jobs) as well as differing transport networks and travel behaviour (e.g. cities have differing levels of transit service as well as populations with differing mode shares). The following are developed and exemplified for measures of access to employment, but can be applied to measures to other types of destinations where there is competition and capacity constraints (e.g. for measuring access to healthcare).

\section{Construction of a Comparative Measure}

The number of people and the number of opportunities in a region is rarely equal. In terms of access to employment, the number of job opportunities rarely equals the size of the labour force within a region. This could be due to workers commuting in and out of the region, unemployed individuals being part of the labour force who are also competing for jobs, people working multiple jobs, or an urban economy with an excess of job opportunities that remain unfilled. The accessibility measures in (3) will not converge given that the total opportunities in the region does not equal the sum of the population who want to access them (i.e. if $\sum P_{i} \neq \sum O_{j}$ ). To allow for convergence, either $O$ or $P$ can be scaled so that $\sum O j=\sum P i$ prior to computing accessibility in (3). However, the quantity and spatial distribution of these imbalances are most likely different when comparing between 
cities, and therefore should be accounted for when generating comparative accessibility measures. So rather than equalizing $P$ or $O$ prior to iterating, we propose that they can be standardized using the mean accessibility of the population observed after the first iteration. This allows $A_{i}$ to be interpretable as an ersatz opportunities per person metric. The equation for $A_{i}$ is updated as 5 follows to incorporate this standardization.

$$
\begin{gathered}
A_{i}=\frac{\bar{A}^{o}}{\bar{A}^{c}} \sum_{j=1}^{J} \frac{O_{j} f\left(t_{i, j}\right)}{L_{j}} \quad L_{j}=\sum_{i=1}^{I} \frac{P_{i} f\left(t_{i, j}\right)}{A_{i}} \\
\bar{A}=\frac{\sum_{i=1}^{I} P_{i} A_{i}}{\sum_{i=1}^{I} P_{i}}
\end{gathered}
$$

$6 \bar{A}^{o}$ is the mean accessibility after the first iteration and $\bar{A}^{c}$ is the mean accessibility after each 7 iteration, $c$. Figure 2 compares three cities of similar urban form, but with different levels of 8 imbalance; one where there is a greater labour force than the number of jobs $\sum P_{i}>\sum O_{j}$ (e.g. 9 due to unemployment), the second where $\sum P_{i}=\sum O_{j}$, and the third where $\sum P_{i}<\sum O_{j}$ (e.g. 10 due to an excess of employment opportunities). These examples are based on the assumption that balance) and travel impedance between polygons is consistent across the plane. These figures indicate how the mean remains stable after each iteration when standardized. The figures also show how as there are more people competing for jobs, the lower the average level of accessibility in the region. Running the same simulation with jobs concentrated in the centre (i.e. a mono-centric urban form) returns similar results, but with a greater range in accessibility from the centre to the periphery. 
Figure 2: Comparing competitive accessibility for three cities with differing imbalance between the population, $P$, and opportunities, $O$

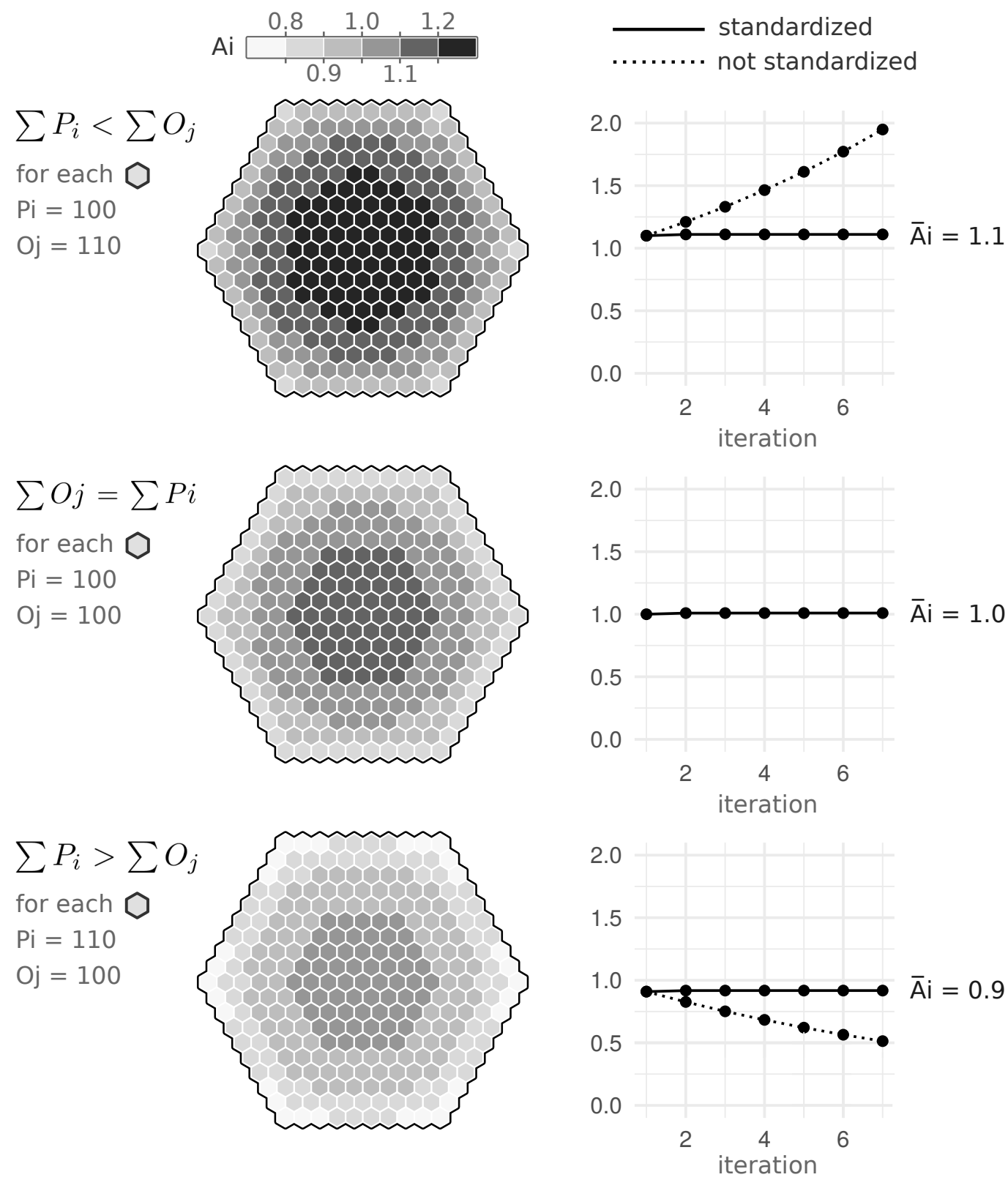

Modal split is another important factor to consider when modelling place-based accessibility. In most cities, people travel to work by different travel modes, and compete for jobs within a multimodal labour force (Shen, 1998; Sanchez, Shen, \& Peng, 2004). For example, a job at $j$ would be more attractive for someone at $i$, if they have regular access to a private vehicle and the commute by car from $i$ to $j$ is faster than the commute by transit. Therefore, we need mode specific measures of $A_{i}$, and we also need to expand the measure of $L_{j}$ to account for multiple modes (e.g. the labour force that can reach $j$ will be a combination of those who travel by transit and car). This can be 
1 accomplished as follows.

$$
A_{i, \lambda}=\frac{\bar{A}^{o}}{\bar{A}^{c}} \sum_{j=1}^{J} \frac{O_{j} f\left(t_{i, j, \lambda}\right)}{L_{j}}, \quad L_{j}=\sum_{\forall \lambda \in \Lambda} \sum_{i=1}^{I} \frac{\alpha_{i, \lambda} P_{i} f\left(t_{i, j, \lambda}\right)}{A_{i, \lambda}}, \quad \sum_{\forall \lambda \in \Lambda} \alpha_{i, \lambda}=1
$$

Figure 3: Example scenario for comparing competitive accessibility between travel modes.

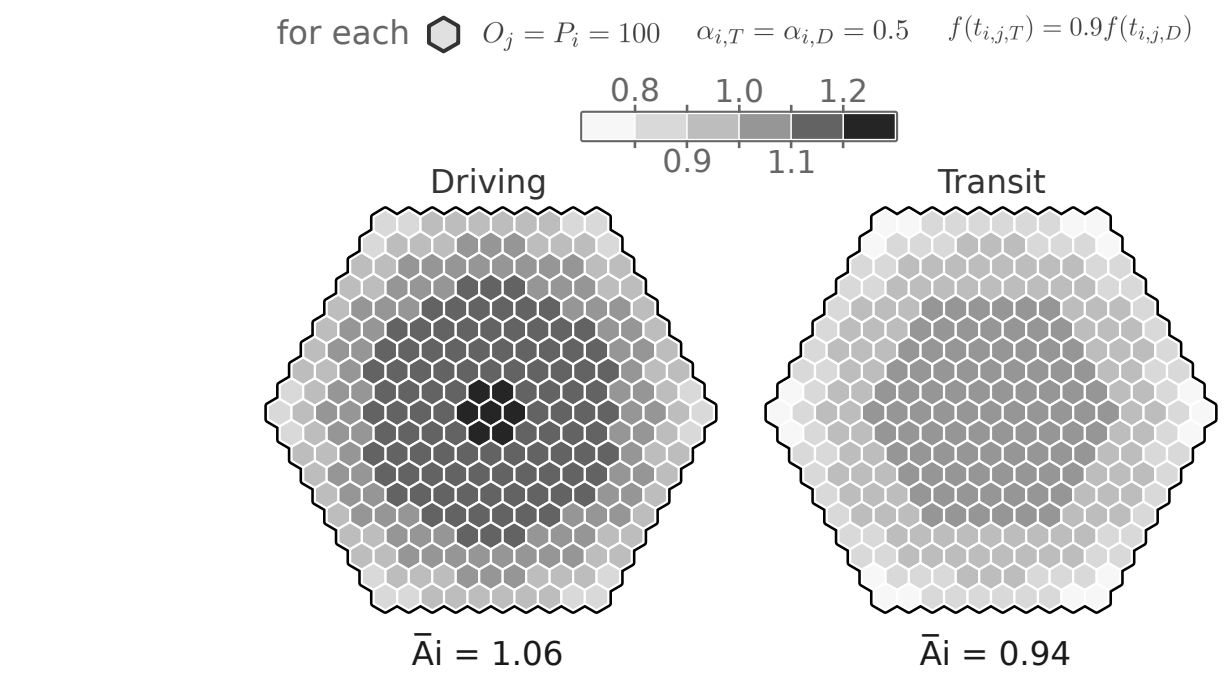

Where $\lambda$ is a travel mode. $\alpha_{i, \lambda}$ is the mode share for travel to work trips of the labour force at location $i$ and $t_{i, j, \lambda}$ is the travel time from $i$ to $j$ for the mode, $\lambda$. The formula for the population mean level of access is updated to account for multiple modes.

$$
\bar{A}=\frac{\sum_{\forall \lambda \in \Lambda} \sum_{i=1}^{I} \alpha_{i, \lambda} P_{i} A_{i, \lambda}}{\sum_{i=1}^{I} P_{i}}
$$

The measures of $A_{i, \lambda}$ now depend on the mobility each mode provides relative to other modes, as well as the mode share for different zones. Figure 3 exemplifies with a case where we assume that the mode share for each zone is $50 \%$ driving and $50 \%$ transit and that the impedance function for travel time to each adjacent zone for transit is $90 \%$ than that by driving (i.e. $f\left(t_{T}\right)=0.9 f\left(t_{D}\right)$ ). Since mode share is equal across the region in this example, the mean accessibility by transit is also $90 \%$ than that by driving.

\section{Computing Access to Employment for Canadian Cities}

The examples in the previous figures are overly simplistic. Real cities have complex transport networks and non-uniform spatial distributions of population, employment, and mode share. Therefore, to further demonstrate the measure presented in (6-7), we compute and compare access to employment for the eight largest urban regions in Canada. By descending order of population, these 
1 are Toronto, Montreal, Vancouver, Calgary, Ottawa, Edmonton, Quebec City, and Winnipeg. For

this analysis, we only make use of open-source data and tools in order for the procedure to be replicated and improved upon with minimal cost. This decision does come with some limitations, as congested travel speeds are not available in any open source datasets that we are aware of.

The boundaries of the urban regions for our analysis are Census Metropolitan Areas (CMA). CMAs are agglomerations of municipalities which pertain to urban areas with a population of over 100,000 in which at least $50 \%$ of the employed labour force works in the region's core, as determined from commuting data from the previous census (Statistics Canada, 2016a). Although imperfect, this measurement provides consistency of what constitutes the boundaries of urban regions across Canada. For our analysis, any adjacent CMAs are merged into a single region due to the commuting flows and transit agencies that link adjacent CMAs.

For the eight regions, we use 2016 census Dissemination Areas (DA) to model the home locations of the labour force. DAs are the smallest areas in which socio-economic data is available from the quinquennial Canadian census, minimizing error due to the modifiable areal unit problem (see Kwan and Weber (2008) for a discussion of MAUP and its effects in accessibility research). DAs are designed and delineated for populations of 400 to 700 persons (Statistics Canada, 2016a), and have been used in other studies on transit accessibility in Canada (Widener et al., 2017; Wessel et al., 2017). Specifically, we use the population weighted centroids of DAs snapped to the closest walking network segment to model the home locations of residents. Larger, neighbourhood sized Census Tracts (CT), however, are used for the location of employment, as they are the smallest geography in which complete employment data was available for the 2016 census. Since different spatial units were used for the origins and destinations, the issue of self-potential does not apply in our study because we are using two different sets of spatial units to model the demand and supply locations. The issue of self-potential appears when the travel time for the demand and supply within an areal unit is zero (Frost \& Spence, 1995). For the few cases where DAs and CTs are the same, a population weighted centroid was used for the origin and the geometric centroid for the destination, so all travel times were greater than zero. It should be noted that several of these urban regions also run their own travel surveys (e.g. the Transportation Tomorrow Survey in the Toronto Region) with home and employment locations of residents, but we required data collected with consistent methodology across the country. Regional travel surveys typically have much more detailed travel diaries, but survey a lower percent of the overall population. The long-form census which we draw our data from is a $25 \%$ representative sample of Canadian households.

Another primary input into our analysis are travel times between where people live and potential places of employment. To compute these travel times, we built custom network graphs for each region. The travel times for driving were computed using the routing engine, Open Source Routing Machine (OSRM) (Luxen \& Vetter, 2011), as it includes detailed consideration for driving attributes like speed limits, turn restrictions, and one-way streets. Due to a lack of open-source network level congestion data, travel times for driving were computed as free-flow speeds using 
OpenStreetMap data, and then multiplied by a congestion factor, $k_{c}$, to account for how peak-hour travel is slower than off-peak. The congestion factors were set at 1.7 for Toronto and Vancouver, 1.6 for Montreal, 1.5 for Ottawa, and 1.4 for the remaining four cities. These values were estimated from reports examining costs of congestion in Canadian cities (Metrolinx, 2008; Urban Transportation Task Force, 2012) as well as from TomTom, which hosts an online worldwide ranking of congestion by city (TomTom, 2018). We also apply a minor two minute penalty for parking, $t_{p}$. The peak hour travel time by driving between two locations, $t_{i, j, d}^{*}$, is thus calculated from the free flow travel time, $t_{i, j, d}$ via $t_{i, j, d}^{*}=k_{c} t_{i, j, d}+t_{p}$. Using commercial speed profile data would likely improve the accuracy of our calculations. However, a secondary objective of our work was to only use data that was open-source and freely available. Research by Salonen and Toivonen (2013) showed that the correlation between travel times computed with and and without modelling link-level congestion were 0.98 , meaning that the relative differences between auto travel times in each region would only have slight variation. Therefore, this is a reasonable input for our accessibility measures which compare auto and transit given that we scale travel times to the mean level of congestion in the region. As well, there may be some spatial error in using a static exogenous parking parameter. Central areas may have increased parking times as there are more people searching for a spot, but conversely peripheral areas have larger surface lots, which can require longer walks from the car to the work location. As well parking time could also be associated with occupation class or income level (e.g. high income workers would be more likely to have a spot in their building of employment, rather using on-street parking). Individual variations in parking time would likely only have a slight effect on resulting accessibility measures since they are aggregated to areal units.

Travel times by transit were computed using the open-source routing engine OpenTripPlanner (2017). These travel times are inclusive of the time walking to and from stops, wait times, invehicle travels times, and transfers. This has two sets of inputs. The first are the walking networks in each of these cities from OpenStreetMap. The second are transit schedules in the form of GTFS (General Transit Feed Specification) data for every transit agency that serves these urban regions, circa May 2016 in order to align with the collection dates of the 2016 census. We use these graphs to compute travel time matrices for each of the eight urban regions in our study. Because of the inherent temporal variations in transit schedules, we follow the precedent in the literature to compute transit travel times for every minute of the morning commute period (Owen \& Levinson, 2015; Farber \& Fu, 2017), to be subsequently averaged when computing accessibility metrics. Although this is common practice in the literature, taking the average may under-estimate accessibility as people are likely to select a time that minimizes their commute. For example, it may make more sense to take the maximum accessibility for 15 minute blocks; but conversely, this may over-estimate accessibility if this is dependent on transfers which may be possible based on the schedule data, but unlikely in reality given congestion and bus-bunching during peak periods. Recent studies have looked at the variation in minute-by-minute accessibility measures (Conway et al., 2018) and comparing schedule versus real-time (e.g. GPS tracked) measures of accessibility (Wessel et al., 2017). However more research is likely needed linking travel behaviour outcomes to 
proper selection of accessibility metrics based on transit schedules. This is not in the scope of our paper, but would be a fruitful direction for future research.

For our analysis, we computed travel times in parallel over several processing units which output results for multiple departure times, $\tau$. The outputs are stored in a three-dimensional array, $T_{i, j, \tau}=\left\{t_{i, j, \tau}\right\}$, where each cell, $t_{i, j, \tau}$, is the travel time from the origin zone, $i$, to the destination zone, $j$, for a specific departure time, $\tau$. Due to heavy computation, travel times were capped at 90 minutes, assuming that no one would be willing to travel to jobs that require more than a 90 minute commute. For our study of Canadian cities, we expand the measure of competitive accessibility presented in (7) to account for a labour force which commutes by car or by transit. This includes averaging transit over the morning commute period (for every minute, $\tau$, from 7:00am to $8: 59 \mathrm{am})$ because of temporal variations in transit schedules.

$$
\begin{gathered}
A_{i, T}=|120|^{-1} \sum_{\tau \in M} \frac{\bar{A}^{o}}{\bar{A}^{c}} \sum_{j=1}^{J} \frac{O_{j} f\left(t_{i, j, \tau}\right)}{L_{j}} \\
A_{i, D}=\frac{\bar{A}^{o}}{\bar{A}^{c}} \sum_{j=1}^{J} \frac{O_{j} f\left(t_{i, j, d}\right)}{L_{j}} \\
\bar{A}=\frac{\sum_{\forall \lambda \in \Lambda} \sum_{i=1}^{I} \alpha_{i, \lambda} P_{i} A_{i}}{\sum_{i=1}^{I} P_{i}} \\
L_{j}=|120|^{-1} \sum_{\tau \in M} \sum_{i=1}^{I} \frac{\alpha_{i, T} P_{i} f\left(t_{i, j, \tau}\right)}{A_{i, T}}+\sum_{i=1}^{I} \frac{\alpha_{i, D} P_{i} f\left(t_{i, j, d}\right)}{A_{i, D}}
\end{gathered}
$$

$A_{i, T}$ is the accessibility measure for transit, and $A_{i, D}$ for driving. $\alpha_{i, D}$ is the commute mode share ratio of workers at location $i$ who travel to work via private vehicle. $\alpha_{i, T}$ is the mode share ratio by transit and walking. The mode share for transit for our study is assumed as the total non-driving commuting population $\left(\alpha_{i, T}=1-\alpha_{i, D}\right)$, and therefore also includes the small percent of those who take active modes (bike or walk). This assumes that those who bike or walk to work are also able to commute to work by transit, but not by car.

We did not have accurate flow data in order to calibrate the travel time impedance functions, a practice that frequently occurs in the literature (e.g. Horner, 2004; Caschili et al., 2015). As an alternative, we use a half-life model specification of distance decay to select an exponential decay function parameterized such that the median commute duration returns a value of 0.5 with a maximum value of 1 at $t_{i, j}=0$ (see Östh et al. (2016) on the use of adopting half-life models for decay functions). 30 minutes is approximately the median commute duration for journey to work trips in Canadian cities (Statistics Canada, 2016b). This results in the following exponential decay function:

$$
f\left(t_{i, j}\right)=e^{-0.0231 t_{i, j}}
$$


For thousands of zones, and minute-by-minute travel times, the process for computing multiple iterations of competitive accessibility is computationally intensive. Therefore, we stopped iterating when the correlation with the previous iteration was $r>0.999$. This level of convergence was reached after 3 or 4 iterations, depending on the city.

The results are summarized by region in Table 1 and Figure 4 . We tabulate data for both transit access and auto access, as well as a ratio between transit and auto access, to examine the differences between these two modes. The complete dataset of accessibility measures, as well as the code used to compute them, are publicly available on GitHub (https://github.com/SAUSyLab/canada-transit-access).

Table 1: Summary of results for each urban region for transit (T) and by auto (D)

\begin{tabular}{lllllllllll}
\hline & & & & \multicolumn{3}{c}{ Mode Share $^{\S}$} & Mean $A_{i}$ & Max $A_{i}$ \\
\cline { 6 - 10 } & Population & Labour Force & Jobs & $\alpha_{D}$ & $\alpha_{T}$ & $\bar{A}_{D}$ & $\bar{A}_{T}$ & $A_{D, \max }$ & $A_{T, \max }$ \\
\hline Toronto & $8,335,444$ & $4,524,570$ & $3,462,100$ & 0.73 & 0.27 & 1.00 & 0.31 & 1.74 & 1.16 \\
Montreal & $4,098,927$ & $2,189,115$ & $1,756,640$ & 0.69 & 0.31 & 1.08 & 0.31 & 1.57 & 0.90 \\
Vancouver & $2,745,461$ & $1,498,535$ & $1,091,405$ & 0.72 & 0.28 & 0.91 & 0.39 & 1.33 & 1.03 \\
Calgary & $1,392,609$ & 816,385 & 587,280 & 0.78 & 0.22 & 0.85 & 0.25 & 1.66 & 0.72 \\
Ottawa & $1,323,783$ & 727,160 & 595,950 & 0.72 & 0.28 & 1.02 & 0.34 & 1.50 & 0.93 \\
Edmonton & $1,321,426$ & 758,150 & 553,660 & 0.83 & 0.17 & 0.84 & 0.21 & 1.15 & 0.66 \\
Quebec City & 800,296 & 437,325 & 375,720 & 0.80 & 0.20 & 0.99 & 0.29 & 1.29 & 0.70 \\
Winnipeg & 778,489 & 424,250 & 344,320 & 0.79 & 0.21 & 0.92 & 0.39 & 1.16 & 0.75 \\
All & $20,796,435$ & $11,375,490$ & $8,767,075$ & 0.74 & 0.26 & 0.98 & 0.31 & 1.74 & 1.16 \\
\hline
\end{tabular}

$\dagger$ Jobs are only those in the region with a "usual place of work" according to the census, while the labour force also includes the unemployed, those who work at home, and those without a fixed place of work. $\S$ Mode share for transit is assumed as the total non-driving commuting population $\left(\alpha_{T}=1-\alpha_{D}\right)$, and therefore also includes the small percent of those who take active modes (bike or walk)

The maximum levels of transit access across the country are observed in central Vancouver and Toronto. Vancouver has a greater average than Toronto however, likely due to Toronto having a greater abundance of suburban areas with low transit access, pulling down its regional average. Montreal is similar in size as Vancouver, but it has a lower mean and maximum level of access by transit. This can be explained by Montreal having less auto congestion (TomTom, 2018), and a greater network of private access highways, which expedite travel by car (i.e. car commuters can compete for more jobs). The mean level of auto access for Montreal is greater than Vancouver and Toronto. In the Montreal and Toronto regions, each internal municipality typically has its own transit agency, resulting in poorer intra-regional travel, while the central transit agency in Vancouver services multiple municipalities (Vancouver, Richmond, Surrey, etc.).

Calgary and Edmonton have the lowest averages of access to jobs, both by transit and car. The urban form of these two cities is more dispersed, and there is greater separation between residential 
and employment areas. There is also a high concentration of employment in low-density suburban business parks which have limited transit service and require long walking times from bus stops to work destinations. The two Albertan cities also had the highest unemployment rates in 2016 compared to the other cities (the unemployment rate was $9.3 \%$ in Calgary and $8.5 \%$ in Edmonton), meaning that there are more people competing for jobs, bringing down the overall levels of access to jobs.

Winnipeg has the highest average level of transit accessibility outside the three largest cities. Winnipeg has fewer peripheral areas with limited transit service, meaning there are fewer areas pulling down its average, and it does not have any internal motorways which would expedite travel by car. As well, from visual inspection, it has a greater spatial mix of jobs and housing inside the city and there is less concentration of employment in suburban business parks. Similar to Winnipeg, Ottawa and Quebec City have a greater mix of jobs and housing than Calgary and Edmonton. However, Ottawa and Quebec City are each bisected by a large river with limited crossings, and different transit agencies operate on either side, limiting accessibility.

\section{Comparison of competitive and non-competitive measures}

In this section, we examine the correlation between competitive accessibility with non-competitive measures of accessibility in order to understand how they could lead to different results and conclusions. Specifically, we compute Pearson correlation coefficients of competitive accessibility comparing with four different types of standard integral measures as in equation (1); the number of jobs reachable within 30, 45, and 60 minutes, as well as the number of jobs reachable weighted using the exponential decay function in equation (12). These correlations are computed for transit accessibility and for auto accessibility and are presented in Table 2.

We find very high correlations between the gravity measures of accessibility and the competitive measure of accessibility within each of the eight cities. It is the relative locations of employment which are the dominant factor in any these accessibility measures. The labour force is more evenly distributed across each region and accounting for the distribution of the labour force does not appear to have a substantial effect in comparing competitive to non-competitive accessibility measures within cities. However, when analyzing all eight cities at the same time, the correlation coefficients decrease by approximately 0.1 (i.e. when the data for all cities are combined into a single table prior to computing correlations instead of computing correlations for each individual region). This shows that for studies analyzing an individual city or region would likely have very minor differences in results using competitive or non-competitive measures of accessibility, but in a multi-city analysis, the competitive measure is controlling for relative sizes of the labour force and job opportunities (e.g. central areas in larger cities have more nearby jobs, but also a larger labour force competing for these jobs).

We also found that transit mode share had a greater correlation with competitive accessibility 
$1(\mathrm{r}=0.82)$, than a non-competitive gravity measure of accessibility $(\mathrm{r}=0.80)$. However, further 2 multivariate analysis would be required to see the effects of competition on mode share, as there 3 are many other land-use and individual-level factors which influence mode share as well.

Table 2: Correlation coefficients between competitive accessibility and four non-competitive accessibility measures, for transit and auto

\begin{tabular}{|c|c|c|c|c|c|c|c|c|}
\hline & \multicolumn{4}{|c|}{ Transit } & \multicolumn{4}{|c|}{ Auto } \\
\hline & $30 \mathrm{~min}$ & $45 \mathrm{~min}$ & $60 \mathrm{~min}$ & decay* & $30 \mathrm{~min}$ & $45 \mathrm{~min}$ & $60 \mathrm{~min}$ & decay* \\
\hline Toronto & 0.62 & 0.85 & 0.94 & 0.97 & 0.93 & 0.92 & 0.79 & 0.96 \\
\hline Montreal & 0.70 & 0.89 & 0.96 & 0.98 & 0.96 & 0.88 & 0.77 & 0.99 \\
\hline Vancouver & 0.77 & 0.91 & 0.96 & 0.98 & 0.95 & 0.89 & 0.74 & 0.99 \\
\hline Calgary & 0.70 & 0.87 & 0.96 & 0.98 & 0.90 & 0.68 & 0.43 & 0.99 \\
\hline Ottawa & 0.73 & 0.89 & 0.97 & 0.98 & 0.94 & 0.81 & 0.61 & 0.99 \\
\hline Edmonton & 0.74 & 0.89 & 0.97 & 0.98 & 0.93 & 0.87 & 0.71 & 0.98 \\
\hline Quebec City & 0.81 & 0.90 & 0.96 & 0.98 & 0.86 & 0.63 & 0.64 & 0.99 \\
\hline Winnipeg & 0.77 & 0.91 & 0.98 & 0.98 & 0.88 & 0.74 & 0.56 & 0.99 \\
\hline All & 0.66 & 0.83 & 0.87 & 0.89 & 0.86 & 0.71 & 0.50 & 0.82 \\
\hline
\end{tabular}

* computed using the exponential decay function in equation (12)

\section{Case Study: Inequalities of Transit Access in Canadian Cities}

5 We exemplify a use case of these measures of competitive accessibility by analyzing the spatial equity of transit access to employment in Canadian cities. Spatial equity can be defined as how evenly a good or service, like transit provision, is distributed among the overall population over space (i.e. this does not consider differences by socio-economic status). Specifically, we compute the Gini coefficient as measure of spatial equity. Delbosc and Currie (2011) and Bertolaccini and Lownes (2013) have used the Gini to examine the inequalities of nearby transit availability, while Welch and Mishra (2013) used the Gini in measuring the inequality pertaining to different aspects of transit connectivity. We use the Gini to measure the inequalities of competitive access to employment. Table 3 indicates the Gini for each region, by transit and by car. The greater the values, the greater amount of inequality of access to employment (the Gini ranges from 0 to 1 ). Table 3 also compares the results of the competitive measure of accessibility with a non-competitive measure, both computed using the decay function in (12). 
Table 3: Gini coefficients for competitive and non-competitive accessibility*

\begin{tabular}{lccccc}
\hline & \multicolumn{2}{c}{ Competitive } & & \multicolumn{2}{c}{ Non-Competitive } \\
\cline { 2 - 3 } \cline { 5 - 6 } & Transit & Car & & Transit & Car \\
\hline Toronto & 0.43 & 0.25 & & 0.54 & 0.35 \\
Montreal & 0.39 & 0.19 & & 0.47 & 0.22 \\
Vancouver & 0.37 & 0.19 & & 0.42 & 0.22 \\
Calgary & 0.29 & 0.11 & & 0.36 & 0.14 \\
Ottawa & 0.34 & 0.17 & & 0.40 & 0.20 \\
Edmonton & 0.36 & 0.12 & & 0.42 & 0.17 \\
Quebec City & 0.31 & 0.11 & & 0.38 & 0.14 \\
Winnipeg & 0.24 & 0.10 & & 0.28 & 0.12 \\
All & 0.40 & 0.21 & & 0.52 & 0.38 \\
\hline
\end{tabular}

* all computed using the exponential decay function in equation (12)

Overall, there are higher values of inequality for Toronto, Montreal, and Vancouver. These regions contain extremely high access neighbourhoods located in their downtown cores, which are within walking distance to major employment centres, as well as rapid and regional transit services linking to other employment areas. The range in access between their centres and peripheries results in greater levels of inequality. Smaller cities tend to have more equal levels of access, but their central areas have lower levels of access than the centres of Toronto, Montreal, and Vancouver. The larger cities also have a greater abundance of low access suburban areas. Out of the mid-size cities, Edmonton and Calgary have greater levels of inequality of transit access, while Winnipeg is the most equitable.

It should be noted that the Gini will change depending on the scale of analysis (Bertolaccini \& Lownes, 2013). For example, if we remove suburban municipalities within the region and only examine the City of Toronto, which has more frequent transit and more transit-oriented development, then the resulting Gini coefficient for competitive transit access reduces from 0.43 to 0.15 as it includes fewer suburban areas with minimal transit service.

Table 3 indicates that using non-competitive measures of accessibility result in greater levels of inequality than competitive measures of accessibility. For the case of Canadian cities, central areas with high employment concentrations also have higher levels of population density nearby who are competing for employment. Central areas typically have lower levels of competitive accessibility than indicated by standard integral measures, while peripheral areas typically have relatively higher access as there are less people competing for nearby jobs. This reduction in the range of accessibility results in lower inequality measures, and not accounting for competition can potentially inflate conclusions of regional transit equity studies.

Table 3 and Figure 4 also highlight how there are substantial disparities by travel mode. Transit accessibility is less than one-third that of auto-accessibility on average. The distribution 
1 of transit access is much more unequal than the distribution of access to jobs by car in each of 2 the eight cities. Transit networks are typically concentrated along certain corridors and are more 3 radially focused compared to regionally dispersed road networks. Many suburban areas have sparse and infrequent transit service, meaning there is a greater share of neighbourhoods at the left of the distributions. Toronto and Vancouver have the greatest overlap of frequency distributions between transit and car. These two cities have high levels of transit accessibility in their centres as well as peripheral communities which are far from the major employment centres which thus have lower levels of auto access. The smaller, more mono-centric cities have less of an overlap between the two travel modes. The gap between transit and auto access also show why suburban areas remain attractive for drivers. When considering competition, especially competition by mode, car drivers in the suburbs are typically doing very well compared to transit users.

\section{${ }_{12} \mathbf{7}$ Conclusion}

In this paper, we expanded measures of competitive access to destinations so that they can be used to accurately compare results both within and between cities and by travel mode. There are three primary contributions to methodology being made. First, this formulation uses an iterative process to account for competition among the labour force for jobs, and among employers for potential employees. Second, it is expanded to account for any regional differences in transportation networks and travel modes, by having parameters for mode share and mode specific travel times between origin and destination locations. And third, it standardizes for any imbalance between the size of the population and the number of opportunities in each region, as these values will vary regionally.

We used this formulation to generate comparative measures of access to employment for eight Canadian urban regions, and then described how access to employment varies between these regions. We find that at a regional level Vancouver and Winnipeg have the highest average levels of transit based access to jobs, and Calgary and Edmonton have the lowest. The neighbourhoods with the maximum levels of transit access are in central Toronto and Vancouver. We then used these measures to examine how access to jobs is distributed within these regions using Gini coefficients. We find that access is more equally distributed in the smaller cities like Winnipeg and Quebec City, while larger urban areas like Toronto, Montreal, and Vancouver have a greater overall inequality of access to employment. Conducting such analysis with standard integral measures can inflate measures of accessibility when comparing between regions, as raw values are not standardized by the size of the labour force or job market. We also found that standard integral measures can potentially over-estimate the extent of spatial inequities of accessibility. 
Figure 4: Frequency distributions of accessibility for each region $($ blue $=$ transit, red $=$ auto)
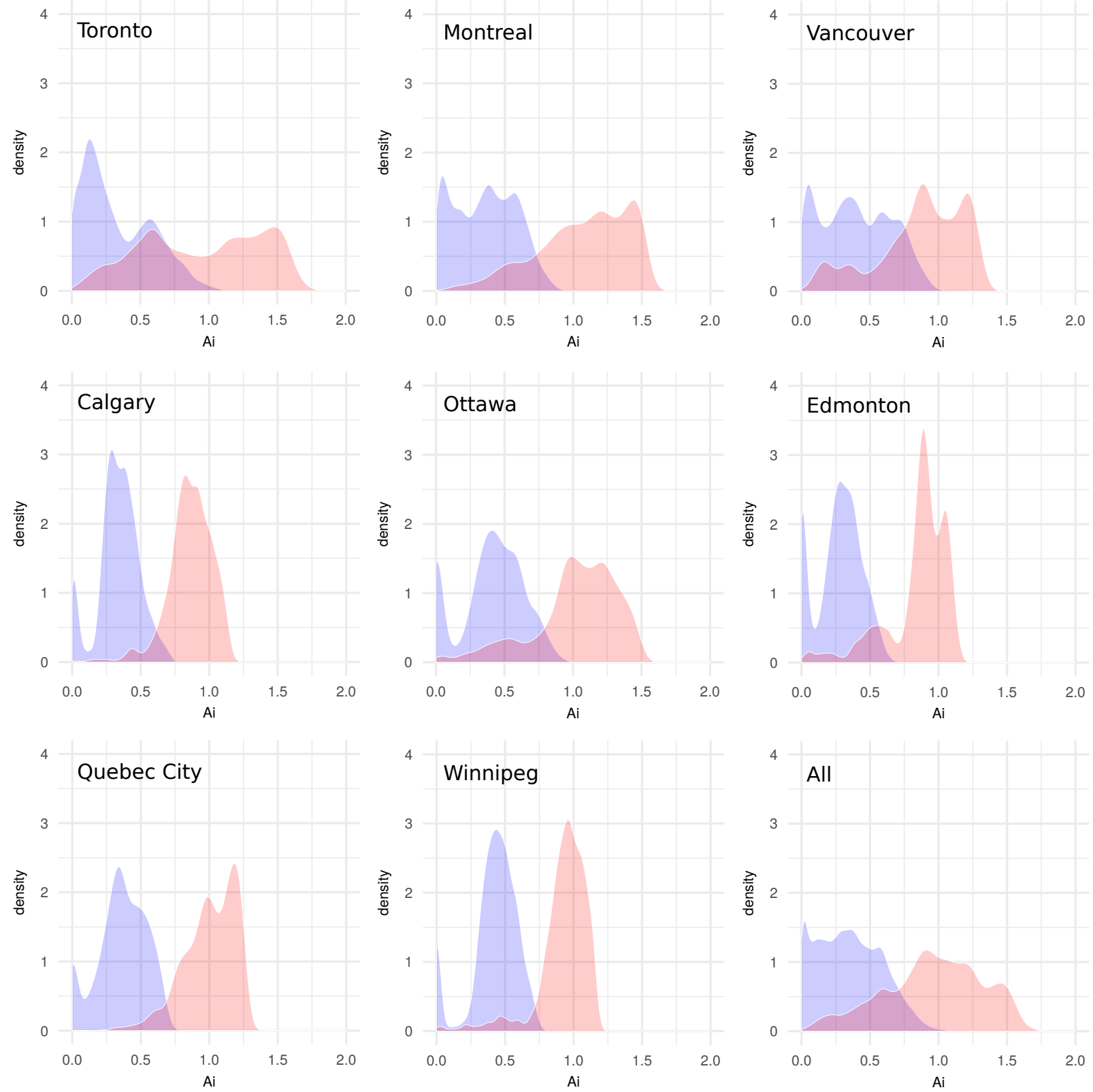

The application of the formulas presented in this paper examine access to all jobs in a region.

2 But certainly, not all workers are competing for the same jobs. One direction for future work is to use the formulations presented in this paper to examine access to employment for specific occupation classes, industry categories, education, or by income levels. This has been applied in other studies analyzing inequalities of accessibility (Cervero et al., 1999; Geurs \& van Eck, 2003; Fan et al., 2012; Fransen et al., 2018). At a simple level, this can be formulated by replacing the number of jobs, $O_{j}$, and size of the labour force, $P_{i}$, by counts for specific sub-groups (e.g. by occupation class 
or income level). However, it is certainly possible that people looking for work may not only be competing for jobs within their specified income bracket or occupation class. For example, those in middle-income brackets may also consider lower income jobs if there is dearth of middle-income jobs available, resulting in greater competition for lower-income jobs as well. Future research should examine how to accurately incorporate weights by job type and account for competition across job categories into the formulas presented in this paper. Similarly, we weighted by zonal mode share to estimate the potential of the labour force to access employment opportunities from each zone. However, individuals may have more than one mode available to them, and the extent to which they compete for employment by different modes will be sensitive to individual ability, resources, and preferences (e.g. whether they have a driver's license, monetary cost of travel with respect to income, or sensitivity to longer walking distances to transit stops). More in depth analysis regarding behavioural impacts on competitive accessibility would likely require individual travel behaviour data, rather than the zone-based census data used in this study. The census data used in this study was also limited to the set of all jobs and population within the region, but the distribution of job seekers and job openings could have differing spatial patterns (Fransen et al., 2018). Data for job openings and job seekers is unavailable Canada-wide. If it were available, it would provide the opportunity for more refined accessibility measures as well as a way to validate existing measures.

In summary, we recommend that the competitive measure outlined in this paper be used instead of more common non-competitive measures in certain cases, specifically for multi-region studies on analyzing access to employment in which values are being directly compared between cities, or as model parameters for analyses predicting behavioural outcomes like mode share, activity participation rates, or unemployment. Our research also shows how competitive measures can be used to highlight modal inequalities in accessibility. Competitive measures could also complement standard non-competitive measures in guiding land-use regulation or transportation investment. For example, an area with high access using a non-competitive integral measure but with relatively lower access in a competitive measure would be a good location to plan for increased employment density, or further improving the links to employment zones. Lastly, since competitive measures are relatively intuitive as they are presented as opportunities per person, they can be useful for communicating results. Effectively communicating the realities of accessibility, through maps or otherwise, is important for providing evidence for urban planning and policy strategies, as well as increasing the understanding of the transport-land use situation to the general public (Geurs \& Van Wee, 2004; Stewart, 2017). 


\section{References}

Benenson, I., Martens, K., Rofé, Y., \& Kwartler, A. (2011). Public transport versus private car gis-based estimation of accessibility applied to the tel aviv metropolitan area. The Annals of Regional Science, 47(3), 499-515.

Bertolaccini, K., \& Lownes, N. (2013). Effects of scale and boundary selection in assessing equity of transit supply distribution. Transportation Research Record: Journal of the Transportation Research Board(2350), 58-64.

Caschili, S., De Montis, A., \& Trogu, D. (2015). Accessibility and rurality indicators for regional development. Computers, Environment and Urban Systems, 49, 98-114.

Cerda, A. (2009). Accessibility: a performance measure for land-use and transportation planning in the Montréal Metropolitan Region (Master's Thesis). McGill University.

Cervero, R., Rood, T., \& Appleyard, B. (1999). Tracking accessibility: employment and housing opportunities in the san francisco bay area. Environment and Planning A, 31(7), 1259-1278.

Conway, M. W., Byrd, A., \& van Eggermond, M. A. (2018). Accounting for uncertainty and variation in accessibility metrics for public transport sketch planning. Journal of Transport and Land Use, 11(1).

Deboosere, R., \& El-Geneidy, A. (2018). Evaluating equity and accessibility to jobs by public transport across canada. Journal of Transport Geography, 73, 54-63.

Delamater, P. L. (2013). Spatial accessibility in suboptimally configured health care systems: a modified two-step floating catchment area (m2sfca) metric. Health 8 place, 24, 30-43.

Delbosc, A., \& Currie, G. (2011). Using lorenz curves to assess public transport equity. Journal of Transport Geography, 19(6), 1252-1259.

De Montis, A., Caschili, S., \& Chessa, A. (2011). Spatial complex network analysis and accessibility indicators: the case of municipal commuting in sardinia, italy. European Journal of Transport and Infrastructure Research, 11(4), 405-419.

El-Geneidy, A., \& Levinson, D. (2011). Place rank: valuing spatial interactions. Networks and Spatial Economics, 11(4), 643-659.

Fan, Y., Guthrie, A. E., \& Levinson, D. (2012). Impact of light rail implementation on labor market accessibility: A transportation equity perspective. The Journal of Transport and Land Use, 5(3), 28-39.

Farber, S., \& Fu, L. (2017). Dynamic public transit accessibility using travel time cubes: Comparing the effects of infrastructure (dis) investments over time. Computers, Environment and Urban Systems, 62, 30-40.

Fotheringham, A. S., \& O'Kelly, M. E. (1989). Spatial interaction models: formulations and applications (Vol. 1). Kluwer Academic Publishers Dordrecht.

Fransen, K., Boussauw, K., Deruyter, G., \& De Maeyer, P. (2018). The relationship between transport disadvantage and employability: Predicting long-term unemployment based on 
job seekers' access to suitable job openings in flanders, belgium. Transportation Research Part A.

Frost, M., \& Spence, N. (1995). The rediscovery of accessibility and economic potential: the critical issue of self-potential. Environment and Planning A, 27(11), 1833-1848.

Geurs, K. T., \& van Eck, J. R. R. (2003). Evaluation of accessibility impacts of land-use scenarios: the implications of job competition, land-use, and infrastructure developments for the netherlands. Environment and Planning B: Planning and Design, 30(1), 69-87.

Geurs, K. T., \& Van Wee, B. (2004). Accessibility evaluation of land-use and transport strategies: review and research directions. Journal of Transport geography, 12(2), 127-140.

Grengs, J., Levine, J., Shen, Q., \& Shen, Q. (2010). Intermetropolitan comparison of transportation accessibility: Sorting out mobility and proximity in san francisco and washington, dc. Journal of Planning Education and Research, 29(4), 427-443.

Handy, S. L., \& Niemeier, D. A. (1997). Measuring accessibility: an exploration of issues and alternatives. Environment and planning A, 29(7), 1175-1194.

Hansen, W. G. (1959). How accessibility shapes land use. Journal of the American Institute of planners, 25(2), 73-76.

Horner, M. W. (2004). Exploring metropolitan accessibility and urban structure. Urban Geography, 25(3), 264-284.

Kawabata, M., \& Shen, Q. (2006). Job accessibility as an indicator of auto-oriented urban structure: a comparison of boston and los angeles with tokyo. Environment and Planning B: Planning and Design, 33(1), 115-130.

Kawabata, M., \& Shen, Q. (2007). Commuting inequality between cars and public transit: The case of the san francisco bay area, 1990-2000. Urban Studies, 44(9), 1759-1780.

Kwan, M.-P. (1998). Space-time and integral measures of individual accessibility: a comparative analysis using a point-based framework. Geographical analysis, 30(3), 191-216.

Kwan, M.-P., \& Weber, J. (2008). Scale and accessibility: Implications for the analysis of land use-travel interaction. Applied Geography, 28(2), 110-123.

Levine, J., Grengs, J., Shen, Q., \& Shen, Q. (2012). Does accessibility require density or speed? a comparison of fast versus close in getting where you want to go in us metropolitan regions. Journal of the American Planning Association, 78(2), 157-172.

Luo, W., \& Wang, F. (2003). Measures of spatial accessibility to health care in a gis environment: synthesis and a case study in the chicago region. Environment and Planning B: Planning and Design, 30(6), 865-884.

Luxen, D., \& Vetter, C. (2011). Real-time routing with OpenStreetMap data. In Proceedings of the 19th acm sigspatial international conference on advances in geographic information systems (pp. 513-516). New York, NY, USA: ACM. doi: 10.1145/2093973.2094062

Merlin, L. A., \& Hu, L. (2017). Does competition matter in measures of job accessibility? explaining employment in los angeles. Journal of Transport Geography, 64, 77-88.

Metrolinx. (2008). Costs of road congestion in the greater toronto and hamilton area: Impact and cost benefit analysis of the metrolinx draft regional transportation plan (Tech. Rep.). 
Miller. (2007). Place-based versus people-based geographic information science. Geography Compass, 1(3), 503-535.

OpenTripPlanner. (2017). (http://www.opentripplanner.org/)

Östh, J. (2011). Introducing a method for the computation of doubly constrained accessibility models in larger datasets. Networks and Spatial Economics, 11(4), 581-620.

Östh, J., Lyhagen, J., \& Reggiani, A. (2016). A new way of determining distance decay parameters in spatial interaction models with application to job accessibility analysis in sweden. European Journal of Transport and Infrastructure Research, 16(2), 344-362.

Owen, A., \& Levinson, D. (2014). Access across america: Transit 2014.

Owen, A., \& Levinson, D. M. (2015). Modeling the commute mode share of transit using continuous accessibility to jobs. Transportation Research Part A: Policy and Practice, 74, $110-122$.

Paez, A., Mercado, R. G., Farber, S., Morency, C., \& Roorda, M. (2009). Mobility and social exclusion in canadian communities: an empirical investigation of opportunity access and deprivation from the perspective of vulnerable groups. Policy Research Directorate Strategic Policy and Research, Toronto.

Salonen, M., \& Toivonen, T. (2013). Modelling travel time in urban networks: comparable measures for private car and public transport. Journal of transport Geography, 31, 143-153.

Sanchez, T. W., Shen, Q., \& Peng, Z.-R. (2004). Transit mobility, jobs access and low-income labour participation in us metropolitan areas. Urban Studies, 41(7), 1313-1331.

Shen, Q. (1998). Location characteristics of inner-city neighborhoods and employment accessibility of low-wage workers. Environment and planning B: Planning and Design, 25(3), 345-365.

Statistics Canada. (2016a). Census dictionary.

Statistics Canada. (2016b). Census of population.

Stewart, A. F. (2017). Mapping transit accessibility: Possibilities for public participation. Transportation Research Part A: Policy and Practice, 104, 150-166.

TomTom. (2018). TomTom Traffic Index. Retrieved from https://www.tomtom.com/en_gb/trafficindex/

Urban Transportation Task Force. (2012). The high cost of congestion in canadian cities (Tech. Rep.). Council of Ministers Responsible for Transportation and Highway Safety.

Weibull, J. W. (1976). An axiomatic approach to the measurement of accessibility. Regional science and urban economics, 6(4), 357-379.

Welch, T. F., \& Mishra, S. (2013). A measure of equity for public transit connectivity. Journal of Transport Geography, 33, 29-41.

Wessel, N., Allen, J., \& Farber, S. (2017). Constructing a routable retrospective transit timetable from a real-time vehicle location feed and gtfs. Journal of Transport Geography, 62, 92-97.

Widener, M. J., Minaker, L., Farber, S., Allen, J., Vitali, B., Coleman, P. C., \& Cook, B. (2017). How do changes in the daily food and transportation environments affect grocery store accessibility? Applied Geography, 83, 46-62. 
1 Wilson, A. G. (1971). A family of spatial interaction models, and associated developments. Environment and Planning A, 3(1), 1-32. 